\title{
Biomolecular interactions of lysosomotropic surfactants with cytochrome $c$ and its effect on the protein conformation: A biophysical approach
}

\author{
Tomasz Janek ${ }^{\mathrm{a}, *}$, Przemysław Czeleń ${ }^{\mathrm{b}}$, Eduardo J. Gudiña ${ }^{\mathrm{c}}$, Lígia R. Rodrigues ${ }^{\mathrm{c}}$, Żaneta Czyżnikowska ${ }^{\mathrm{d}}$ \\ a Department of Biotechnology and Food Microbiology, Wrocław University of Environmental and Life Sciences, 51-630 Wrocław, Poland \\ b Department of Physical Chemistry, Faculty of Pharmacy, Collegium Medium, Nicolaus Copernicus University, 85-950 Bydgoszcz, Poland \\ c Centre of Biological Engineering, University of Minho, 4710-057 Braga, Portugal \\ d Department of Inorganic Chemistry, Faculty of Pharmacy, Wroclaw Medical University, 50-556 Wrocław, Poland
}

\section{A R T I C L E I N F O}

\section{Article history:}

Received 27 November 2018

Received in revised form 19 December 2018

Accepted 5 January 2019

Available online 06 January 2019

\section{Keywords:}

Lysosomotropic surfactant

Cytochrome $c$

Fluorescence quenching

Circular dichroism

Molecular dynamic simulations

\begin{abstract}
A B S T R A C T
The molecular interactions between two single-chain lysosomotropic surfactants DMM-11 (2-Dodecanoyloxyethyl) trimethylammonium bromide) and DMPM-11 (2-Dodecanoyloxypropyl)trimethylammonium bromide) with a small heme-protein (cytochrome $c(\mathrm{cyt}-\mathrm{c})$ ) in Hepes buffer $(\mathrm{pH}=7.4)$ were extensively investigated by surface tension, dynamic light scattering (DLS), circular dichroism (CD) and fluorescence spectroscopy in combination with molecular dynamic simulation techniques. The results demonstrated that surfactants can destroy the hydrophobic cavity of cyt-c, make the $\alpha$-helical become loose and convert it into the $\beta$-sheet structure. The interactions between surfactants and cyt-c are mainly hydrophobic. Molecular modelling approaches were also used to gather a deeper insight on the binding of lysosomotropic surfactants with cyt-c and the in silico results were found to be in good agreement with the experimental ones. This study provides a molecular basis for the applications of proteinsurfactant complexes in biological, food, pharmaceutical, industrial and cosmetic systems.
\end{abstract}

(c) 2019 Elsevier B.V. All rights reserved.

\section{Introduction}

Interactions between proteins and surfactants have been studied for many years [1-6], not only due to their fascinating structural organization, but also to their potential technological applications in biosciences, drug delivery, medicine and food industry. The surfactant-protein complexes become more hydrophilic than either the surfactant or the protein themselves, and the effective increase of the complexes solubility avoids the formation of higher order aggregates [7]. Mostly, electrostatic interactions and hydrophobic associations are the two main driving forces that contribute for the surfactant-protein interactions [8,9]. Likewise, surfactants can cause protein conformational changes leading to the protein folding or unfolding depending on the concentrations of both surfactants and proteins. Several biophysical methods such as isothermal titration calorimetry [4], surface tension analysis [10], fluorescence [11] and circular dichroism spectroscopy [12] have been used to unravel the interactions between surfactants and proteins. Parray et al. [13] reported the interactions between cationic gemini surfactant and its monomeric counterpart with phospholipase $A_{2}$, and the results indicated that the tuning of the protein conformations by surfactants

\footnotetext{
* Corresponding author at: Department of Biotechnology and Food Microbiology, Wrocław University of Environmental and Life Sciences, 51-630 Wrocław, Poland E-mail address: tomasz.janek@upwr.edu.pl (T. Janek).
}

changes according to the structure of the surfactants used. Janek et al. [4] studied the interactions between bovine serum albumin (BSA) and cationic quaternary ammonium surfactants (QACs) using the synchronous fluorescence method, and the experiments showed that surfactants mainly interacted with the tryptophan residues of BSA. Hu et al. [14] estimated the Stern-Volmer quenching constants $K_{S V}$ and the corresponding thermodynamic parameters $\Delta H, \Delta G$ and $\Delta S$ of the interactions between BSA and surfactants by the fluorescence quenching method. It was noted that the hydrophobic forces are the predominant intermolecular forces between BSA and the surfactant.

An interesting group of lysosomotropic surfactants are the cationic QACs which, according to their amphiphilic nature, can partition to the phospholipid bilayer and translocate across membranes as uncharged molecules [6]. In contrast to other surfactants that kill cells by acting at the plasma membrane, lysosomotropic surfactants primarily act on within the lysosomes [15]. Several soft cationic QACs showed biological activities against several pathogenic bacterial strains [16], filamentous fungi [17] and also some human tumor cell lines [18]. Therefore, soft cationic QACs can potentially be used in lysosometargeting anti-cancer drugs [19-21]. Cationic QACs can enter the lysosome and possibly induce conformational changes of the proteins involved in apoptosis [22].

In our experiments we used cytochrome $c(\mathrm{cyt}-c)$ as a model protein for various interactions. Cyt- $c$ is a kind of iron-containing metalloprotein 
present in the cells mitochondria. It is a small globular protein (molecular weight $12,327 \mathrm{Da}$ ) well known for its role in the biological electron transport functions. In horse heart cyt- $c$, the heme group is bond to four amino acids and it is also connected to a noncovalent side-chain, thus making the internal structure around the heme macrocycle relatively rigid [23]. The alpha helix ( $\alpha$-helix) is the dominant structure of cyt-c which is connected by short non-helical segments [23]. Structural alterations are not only important for electron transfer, but also for events like apoptosis [24]. It has been reported that during apoptosis cyt-c undergoes a conformational change, which may be required for binding to Apaf-1 [25].

Herein, we attempted to unravel the nature of the interactions between cyt-c and single-chain quaternary ammonium surfactants in Hepes buffer at physiological $\mathrm{pH}=7.4$ using physicochemical and fluorescence spectroscopic techniques. Moreover, the experimental results were supported by molecular dynamic simulations. Studies on the molecular interactions between DMM-11 or DMPM-11 and cyt-c are helpful to further understand the effects of lysosomotropic surfactants on cyt-c and their biological properties.

\section{Experimental section}

\subsection{Materials}

The surfactants used were quaternary ammonium salts from the esterquats group, namely (2-Dodecanoyloxyethyl)trimethylammonium bromide (DMM-11) and (2-Dodecanoyloxypropyl)trimethylammonium bromide (DMPM-11). The synthesis of these lysosomotropic surfactants was described in detail in earlier studies [26,27]. These surfactants were recrystallized three times and characterized by electrospray ionization mass spectrometry (ESI-MS) and nuclear magnetic resonance ( ${ }^{1} \mathrm{H}$ NMR) spectroscopy (Table S1). The chemical structures of these surfactants are illustrated in Fig. S1. All reagents and solvents were of the highest available grade and were used as received. Horse heart cytochrome $c$ (cyt-c), 4-(2-Hydroxyethyl)piperazine-1-ethanesulfonic acid sodium salt (Hepes) and sodium chloride were purchased from Sigma Chemical Co. (St. Louis, MO). Stock solutions of cyt-c and single-chain surfactants, prepared in Hepes buffer solutions (5 mM Hepes, $150 \mathrm{mM} \mathrm{NaCl}$, pH 7.4) were further used to prepare samples with the desired concentrations. The concentration of the cyt- $c$ was determined spectrophotometrically at $410 \mathrm{~nm}$ using an $\varepsilon=106,100 \mathrm{M}^{-1} \mathrm{~cm}^{-1}$. All experiments were performed at $25^{\circ} \mathrm{C}$.

\subsection{Surface tension measurements}

Surface tensions $(\gamma)$ were measured using a Krüss K100 Tensiometer (Krüss $\mathrm{GmbH}$, Hamburg, Germany) at $25^{\circ} \mathrm{C}$, according to the du Noüy's ring method [28]. Surfactants and cyt-c were dissolved in Hepes buffer and mixed to obtain several mixtures containing a constant cyt-c concentration ( $1 \mathrm{~g} \mathrm{~L}^{-1}$ ) but different DMM-11 or DMPM11 concentrations. Millipore water was used to calibrate the tensiometer. The platinum ring was thoroughly cleaned with Millipore water between measurements. The surfactants $\gamma$ values in the presence or absence of cyt-c were measured by mixing the adequate stock solutions. The average $\gamma$ values were obtained by measuring each sample in triplicate.

\subsection{Dynamic light scattering (DLS)}

DLS measurements were carried out using a Zetasizer Nano-ZS from Malvern Instruments (UK) to measure the hydrodynamic radii $\left(R_{\mathrm{h}}\right)$ of the cyt- $c$ (with and without surfactants) at $25^{\circ} \mathrm{C}$. Before the measurements, the samples were filtered through a $0.2 \mu \mathrm{m}$ pore size filter directly into the optical cell to remove any impurity. For each sample nine measurements were made with an acquisition time of $20 \mathrm{~s}$. The $R_{\mathrm{h}}$ values were estimated on the basis of an autocorrelation analysis of scattered light intensity data based on translation diffusion coefficient by Stokes-Einstein relationship [29]:

$D=\frac{K_{\mathrm{B}} T}{3 \pi \eta d}$

where $D$ is the diffusion coefficient, $K_{\mathrm{B}}$ is Boltzmann constant, $T$ is the temperature, $\eta$ is the viscosity of buffer solution and $d$ is diameter.

\subsection{UV-visible absorption measurement}

The UV-visible absorption spectra were recorded on a Varian Carry 50 Bio spectrophotometer (Varian, USA) using the $1 \mathrm{~cm}$ path length quartz cell at $25^{\circ} \mathrm{C}$, with the wavelength scanning from $300 \mathrm{~nm}$ to $700 \mathrm{~nm}$.

\subsection{Fluorescence measurements}

The fluorescence spectra were generated with a Varian Cary eclipse fluorescence spectrophotometer using a $1 \mathrm{~cm}$ quartz cuvette. The emission spectra were recorded in the wavelength range of $310-340 \mathrm{~nm}$ by exciting protein at $295 \mathrm{~nm}$ using excitation and emission slit width of $10 \mathrm{~nm}$. All measurements were conducted at $25^{\circ} \mathrm{C}$. To eliminate the inner filter, the fluorescence intensity was corrected using the following relationship [30]:

$F_{\text {cor }}=F_{\text {obs }} \times 10^{\left(A_{\mathrm{ex}}+A_{\mathrm{em}}\right) / 2}$

where $F_{\text {cor }}$ and $F_{\text {obs }}$ are the fluorescence intensity corrected and observed, respectively, and $A_{\mathrm{ex}}$ and $A_{\mathrm{em}}$ are the absorption of the system at excitation and emission wavelengths, respectively.

\subsection{Circular dichroism $(C D)$ measurements}

The CD spectra of cyt-c in the absence and presence of surfactants were performed using a Jasco model J-1500 spectropolarimeter, and were recorded at $25{ }^{\circ} \mathrm{C}$ with a $1 \mathrm{~mm}$ path length cuvette in the wavelength range of 200-260 $\mathrm{nm}$. A scan speed of $50 \mathrm{~nm} \mathrm{~min}^{-1}$ and a response time of $1 \mathrm{~s}$ were used. The spectrum of Hepes buffer was subtracted from the sample spectra for data analysis. The $\beta$-sheet contents were calculated using the Jasco spectropolarimeter software (JWSSE-513 protein secondary structural analysis program).

\subsection{Molecular dynamics simulations}

The molecular dynamics (MD) simulations were performed with version 14 of the AMBER simulation package [31]. The three dimensional structure of horse cytochrome complex used during simulation was obtained from the Brookhaven Protein Database PDB (PDB ID: 1HRC) [32]. The structures of DMM-11 and DMPM-11 surfactants were described with use of AMBER force field parameters. The atomic charges were calculated according to the Merz-Kollmann scheme via the Restrained Electrostatic Potential procedure (RESP) at HF/6-31G* level of theory [33]. In the case of the heme molecule it was used parameterization deposited in AMBER parameter database [34]. The number of surfactant molecules used during preparation of the systems corresponds to CMC obtained from experimental measurements. To setup the surfactant/cyt-c system, we used 8 molecules of surfactants (below CMC) and 30 molecules of surfactants (above CMC). All systems were neutralized by adding sodium cations and were immersed into the TIP3P water box. The MD simulations were performed according to the following criteria: all considered systems after two stage minimization were heated up to 25 during 100 ps of initial molecular dynamics simulation run. Density of systems was equilibrated during next 500 ps of simulation. All initial steps were performed with weak constrains for protein and heme molecules. The first initial stage was followed by 
next 200 ns of MD simulation run. Structural analysis including root mean square deviation (RMSD) and identification of hydrogen bonds were performed using the VMD package [35]. The analysis of hydrogen bonds included three parameters: distance between donor (D) and acceptor atoms $(\mathrm{A})<3.5 \AA$, distance $\mathrm{H}-\mathrm{A}<3 \AA$ and angle $\mathrm{D}-\mathrm{H}-\mathrm{A}>90^{\circ}$. The identification of atomic contacts between protein and surfactant molecules was performed using the ccptraj module implemented in AmberTools 14 package. The solvent accessible surface area (SASA) and solvent accessible volume (SAV) were calculated using the CASTp application [36], the radius of probe sphere was equal to $1.4 \AA$.

\section{Results and discussion}

\subsection{Surface tension and DLS studies}

In order to understand protein-surfactant interactions, the surfactants micellization process in aqueous solution is required. For that purpose, surface tensions were measured and the surfactants critical micellar concentrations (CMC) were determined. The CMC values have been taken from the break points in the surfactant-cyt-c systems (Fig. 1). In the case of DMM-11 and DMPM-11 without cyt-c, typical CMC values can be observed as the saturation of the interface is reached. The calculated values for the CMC of DMM-11 and DMPM11 in Hepes buffer solutions from their surface tension isotherms were $0.75 \mathrm{mmol} \mathrm{L}^{-1}$ and $0.6 \mathrm{mmol} \mathrm{L}{ }^{-1}$, respectively. These CMC values are in good agreement with the ranges previously reported [4]. The observed surface tension of DMM-11 and DMPM-11 solutions containing cyt-c is lower than the values obtained for the pure surfactants due to the surface active nature of cyt-c. It can be seen from Fig. 1 that the surface tension curves corresponding to the surfactant/cyt-c complexes exhibit one break point which marks the concentration above which the aggregation of surfactants occurs. The DMM-11 and DMPM-11 complexed with cyt-c showed CMC values of $1.48 \mathrm{mmol} \mathrm{L}^{-1}$ and $0.75 \mathrm{mmol} \mathrm{L}^{-1}$, respectively. An estimation of the number of surfactant molecules bound to the protein was done under these conditions (i.e. at the $\mathrm{CMC}$ ). We performed the calculation of the surfactant:protein ratios for both systems. The molar ratios estimated in this way were 9:1 for both DMM-11 and DMPM-11 in the presence of cyt-c. These results suggest that both surfactants can interact with cyt-c through electrostatic and hydrophobic forces. These results are similar to those reported for the fluorescence intensity changes of BSA with QACs. Besides, they are also similar to the results reported for the interactions between gelatin and cationic gemini surfactant [37]. On the other hand, Nishikido et al. [38] didn't find significant changes in $\mathrm{CMC}$ using lysozyme and surfactant mixtures at the protein concentration range studied.

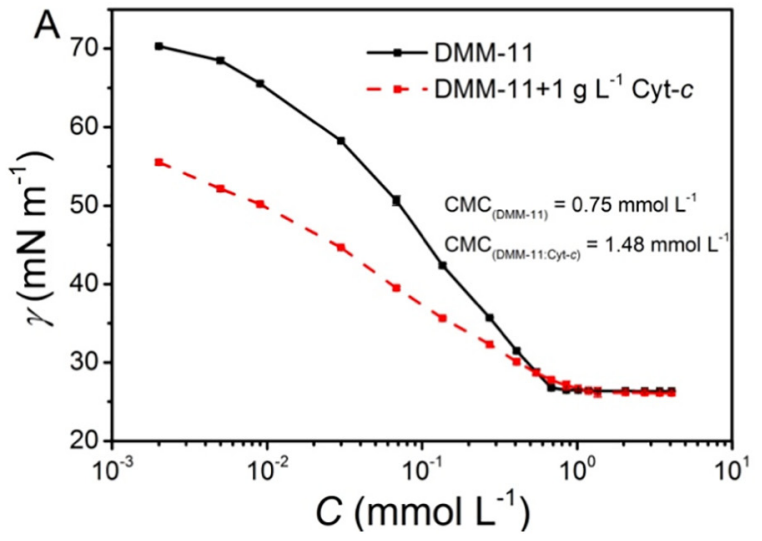

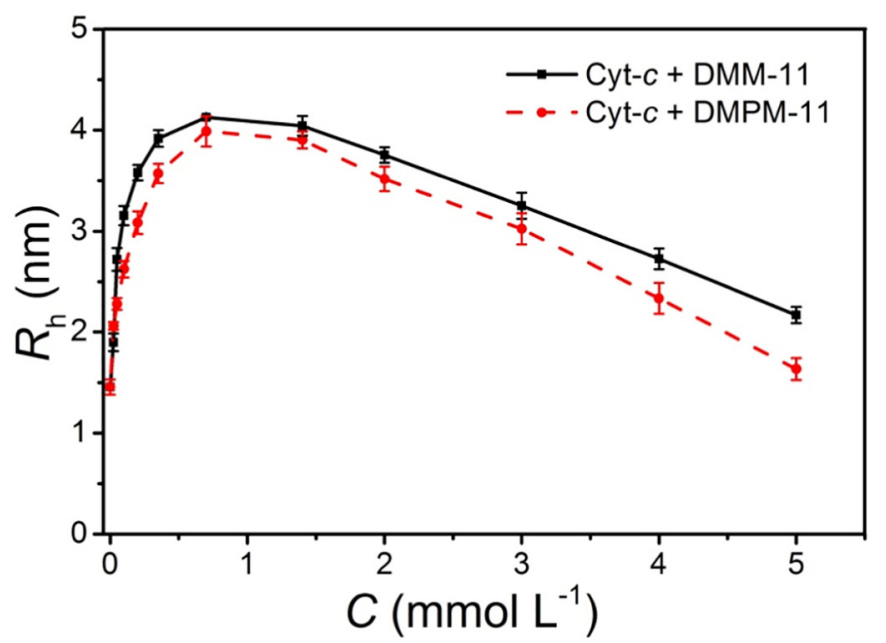

Fig. 2. Effect of surfactants concentrations on the hydrodynamic radius $\left(R_{\mathrm{h}}\right)$ of cyt-c $\left(1 \mathrm{~g} \mathrm{~L}^{-1}\right)$ in Hepes buffer $(\mathrm{pH} 7.4)$ at $25^{\circ} \mathrm{C}$. Results represent the mean of 9 replicates $\pm S D$.

DLS was used to calculate the hydrodynamic radius of the complexes surfactant-cyt-c. In Fig. 2, the calculated hydrodynamic radius $\left(R_{\mathrm{h}}\right)$ was plotted as a function of the surfactants concentration (cyt-c concentration was kept constant). As it can be observed, the $R_{\mathrm{h}}$ of the complexes increased at low concentrations (below the CMC) of DMM-11 and DMPM-11, from $1.45 \mathrm{~nm}$ to 4.12 and $3.98 \mathrm{~nm}$, respectively. Above the $\mathrm{CMC}$, the $R_{\mathrm{h}}$ of cyt-c decreased from $\sim 4 \mathrm{~nm}$ to 2.17 and $1.63 \mathrm{~nm}$ with increasing DMM-11 and DMPM-11 concentrations, respectively. The distinct $R_{\mathrm{h}}$ obtained for the different surfactant/cyt-c complexes can be attributed to the difference in the binding number of cationic surfactants to cyt-c. An increase in the $R_{\mathrm{h}}$ was observed with the addition of a sub-micellar concentration of surfactants. Surprisingly, the $R_{\mathrm{h}}$ decreases upon the addition of a post-micellar concentration of surfactants. This fact correlates very well with the observation that when DMM-11 and DMPM-11 are added to the solutions they tend to interact with native cyt-c to unfold and fold it. The polydispersity index was always $<0.2$ in all experiments.

\subsection{UV-Vis and fluorescence spectra}

A Soret band with a maximum intensity at $410 \mathrm{~nm}$ is typical of the native state of cyt- $c$, indicative of the presence of axial histidine and methionine ligands. The change in the absorbance of cyt-c at $410 \mathrm{~nm}$ was registered in the presence of cationic surfactants and is shown in Fig. S2. The Soret maximum in the presence of DMM-11 and DMPM-

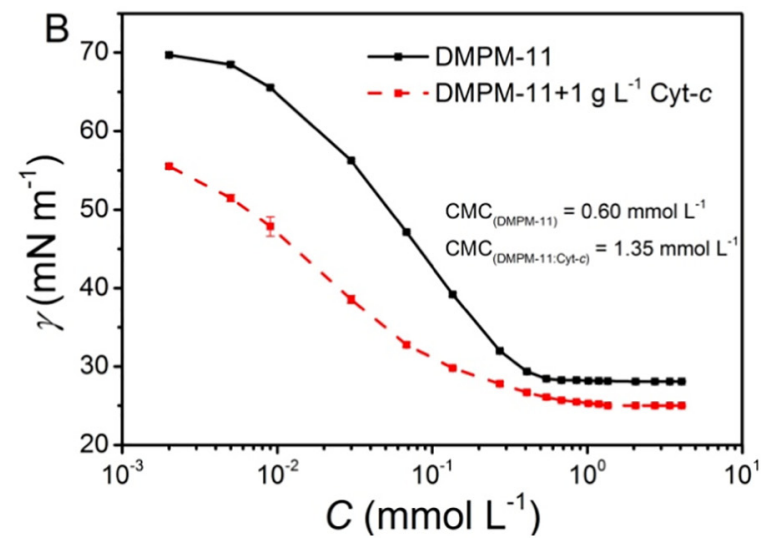

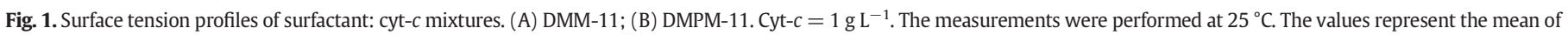
triplicates \pm SD. 
11 remains fixed at $410 \mathrm{~nm}$ over the entire surfactant concentration range investigated, thus indicating the persistence of methionine ligation in cyt-c. A linear increase in the absorbance of the Soret band was observed with the addition of a sub-micellar concentration of the surfactants. Above the CMC, the absorbance decreases with increasing DMM-11 and DMPM-11 concentrations. Prior to CMC, the free surfactants molecules bind to the cyt- $c$ chains, whereas beyond CMC the surfactant/cyt-c micelle interactions prevail. These results were further confirmed by dynamic light scattering assays (Fig. 2).

Horse heart cyt-c contains a single tryptophan residue (Trp59) which is bonded to one of the propionic groups of heme. For the native cyt-c the fluorescence signal is weak because of the proximity of Trp59 to the heme group [39]. The increase in the Trp59 distance from the heme leads to an increase in the fluorescence intensity [40]. Hence, the change in fluorescence intensity is often used as a reliable marker of molecular expansion around the heme. As the excitation wavelength was set to $295 \mathrm{~nm}$, the intrinsic fluorescence of protein can be emitted due to the Trp residue [41]. As shown in Fig. 3, the intrinsic fluorescence intensity of cyt- $c$ is enhanced with the increase of both DMM-11 and DMPM-11 surfactant concentrations, which is ascribed to the fact that both electrostatic and hydrophobic interactions occur as the negatively charged amino acid residues of the protein bind to the positively charged head groups of surfactants, and the hydrophobic chains of the surfactants interact with the adjacent hydrophobic regions of cyt-c. Both cationic surfactants, DMM-11 and DMPM-11, exhibit a similar behavior. Trp exists in the vicinity of the hydrophobic cavity of cyt-c, and
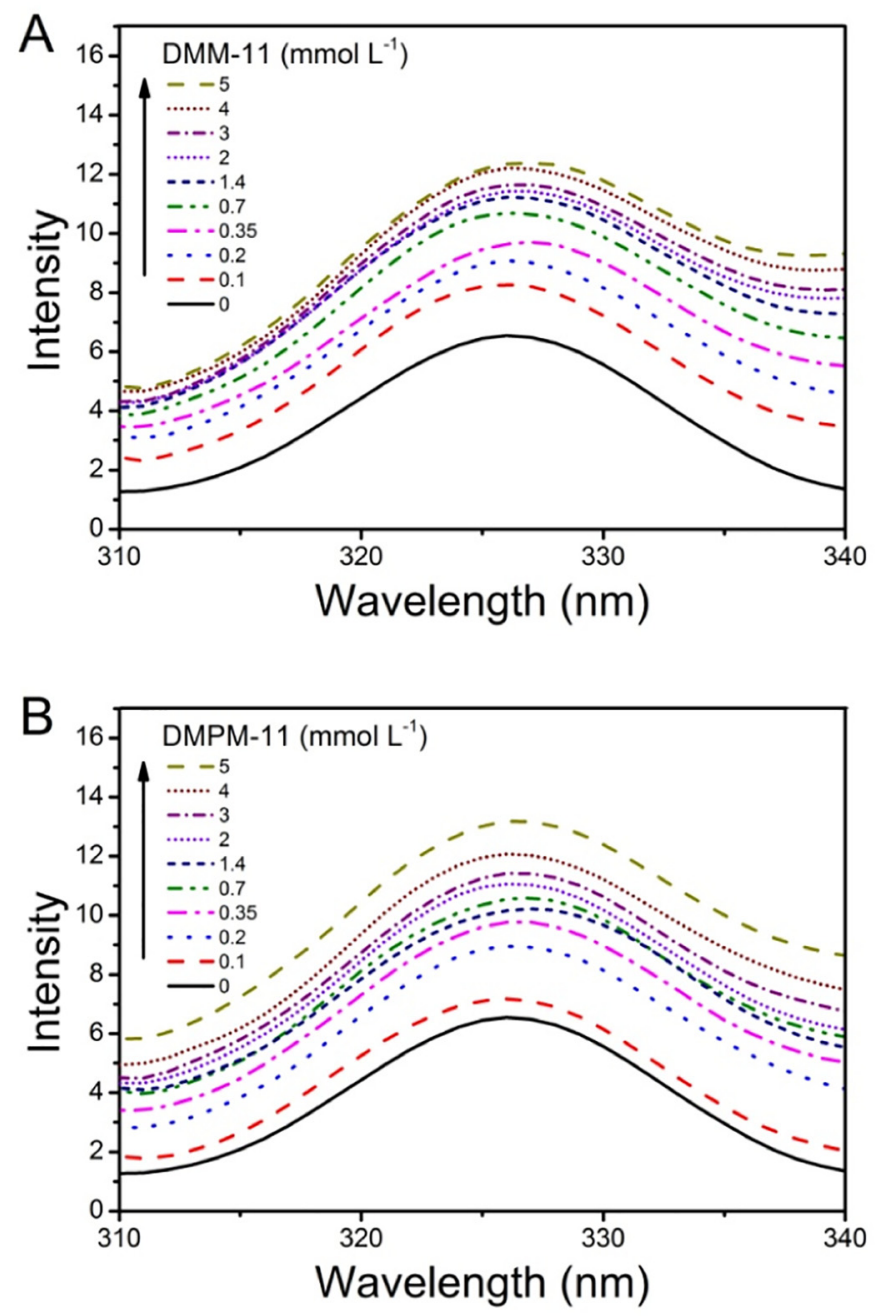

Fig. 3. Fluorescence emission spectra of cyt-c $\left(10 \mu \mathrm{mol} \mathrm{L}^{-1}\right)$ in the presence of different concentrations of DMM-11 (A) and DMPM-11 (B) at $25{ }^{\circ} \mathrm{C}$. the hemichrome in the hydrophobic cavity has a strong quenching effect on the fluorescence of Trp. As described in Fig. 3, the fluorescence of cyt-c is mainly due to Trp59, which is quenched by the heme group through foster energy transfer, thus native cyt-c produces very weak fluorescence. The fluorescence emission in native cyt-c by tryptophan can be seen at $326 \mathrm{~nm}$ with very low intensity due to quenching by the heme group. The DMM-11 and DMPM-11 insert into the hydrophobic cavity of cyt- $c$, forces the hemichrome release and inhibits the quenching of fluorescence. On the other hand, our results suggest that the interaction with the surfactants induced an increase of the samples turbidity and cyt- $c$ unfolding, thus resulting in a conformational change of the metalloprotein with increasing cationic surfactants concentrations. These results are similar to those reported for the fluorescence intensity changes of cyt- $c$ with anionic surfactants [42]. Furthermore, Ahluwalia and co-workers [42] observed that the fluorescence intensity increases significantly with the increase of sodium dodecyl sulfate (SDS) concentrations. A similar trend has been reported for long-chain imidazolium ionic liquid surfactants with cyt-c [12].

\section{3. $C D$ measurements}

CD spectroscopy has been employed as a descent technique to examine the conformational changes of proteins and peptides in aqueous solutions. Therefore, it was used to assess the effect of the DMM-11 and DMPM-11 on the secondary structure of cyt- $c$ Fig. 4 shows the change of far-UV CD spectra of cyt- $c$ at different surfactant concentrations. The CD spectra of cyt-c exhibit two negative bands in the region of 208 and
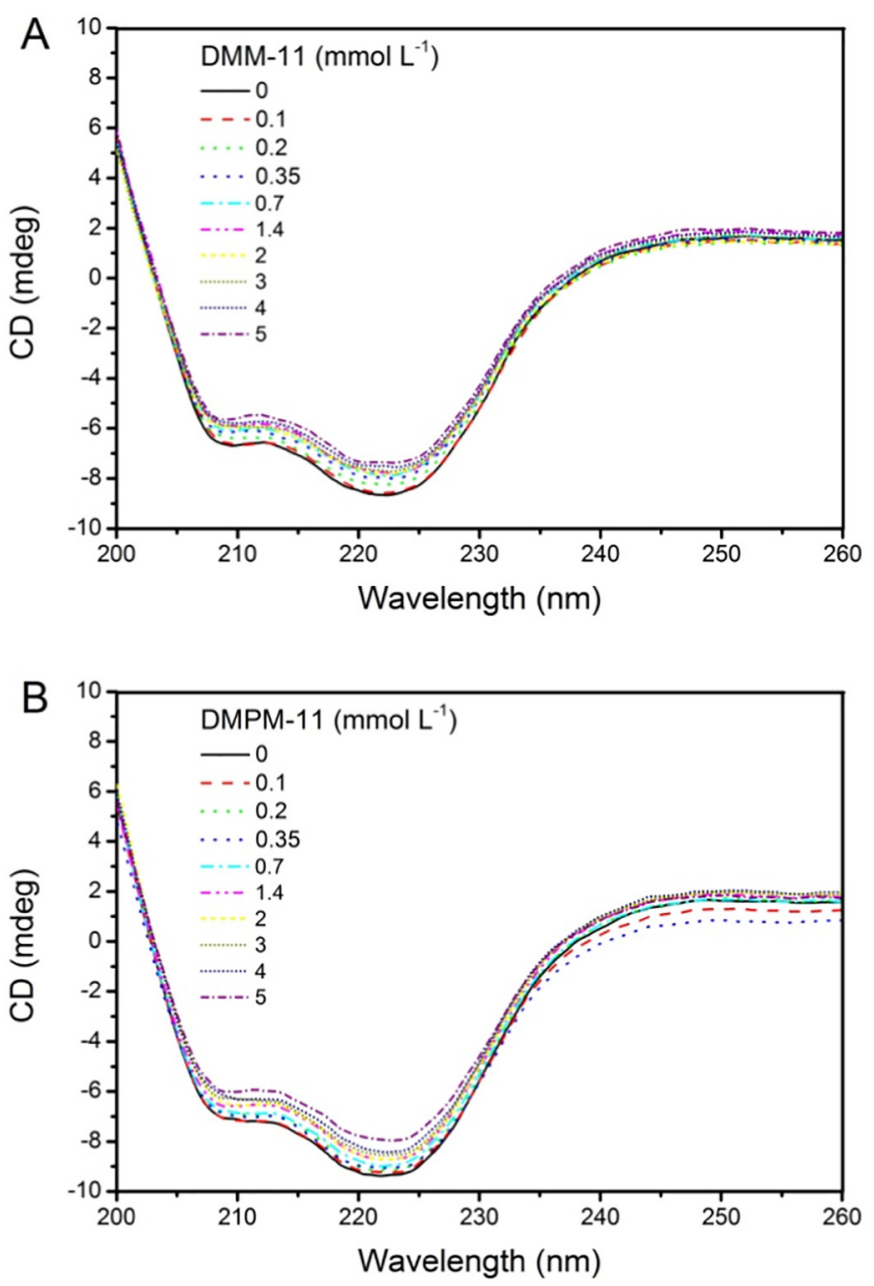

Fig. 4. CD spectra of cyt-c $\left(0.5 \mathrm{~g} \mathrm{~L}^{-1}\right)$ in the absence and presence of DMM-11 (A) and DMPM-11 (B) at $25^{\circ} \mathrm{C}$. 
$222 \mathrm{~nm}$, which correspond to the characteristic peaks of $\alpha$-helical structure [43]. With the increase of surfactants concentrations, the intensity of $C D$ spectra is decreased. The $C D$ results are expressed in terms of mean residue ellipticity (MRE) in $\theta$ or deg $\mathrm{cm}^{2} \mathrm{dmol}^{-1}$ [44]:

$M R E_{208}=\frac{C D_{\text {observed }}}{C n l}$

where $C D_{\text {observed }}$ is the $C D$ value in $C D$ spectrum (mdeg); $C$ is the concentration of cyt-c $\left(4 \times 10^{-5} \mathrm{~mol} \mathrm{~L}^{-1}\right)$; $\mathrm{n}$ is the number of amino acid residues, $n=104 ; 1$ is the path length $(0.1 \mathrm{~cm})$. Afterwards, the $\alpha$ helix content of cyt-c is determined by Eq. (3):

$\alpha-$ helix $(\%)=\left(\frac{-M R E_{208}-4000}{33,000-4000}\right) \times 100 \%$

where $M R E_{208}$ is the value of MRE at $208 \mathrm{~nm} ; 33,000$ is for the random coil and $\beta$-form conformation and 4000 is the MRE value of a pure $\alpha$ helix at $208 \mathrm{~nm}$. With the increase of DMM-11 or DMPM-11 concentrations, the $\alpha$-helicity of cyt-c was changed. CD spectra of free cyt-c at $25^{\circ} \mathrm{C}$ yielded a $42.8 \% \alpha$-helical structure. The addition of $5 \mathrm{mmol} \mathrm{L}^{-1}$ of DMM11 or DMPM-11 to cyt-c caused a conformational modification of the cyt-c to $32.1 \% \alpha$-helical and $34.0 \% \alpha$-helical, respectively. The results clearly show that the increase of surfactant concentrations leads to a decrease of the $\alpha$-helical contents of cyt-c, thus indicating that $\alpha$-helixes become loose and are converted into the $\beta$-sheet structure (Table S2). In contrast, Singh and co-workers [12] reported an opposite trend when studying the interaction of a long-chain imidazolium ionic liquid surfactants with cyt-c.

\subsection{Computational analysis of surfactant-protein interactions}

The structure of the cyt-c system analyzed in the molecular dynamics stage is illustrated in Fig. S3. Both geometries from first and 200 ns exhibit high similarity suggesting the structural and dynamic stability of this system. This was further confirmed by the RMSD values determined for cyt-c and heme with and without surfactants over the whole 200 ns simulation time (Fig. S4). The presence of either DMM11 or DMPM-11 led to the fluctuation of protein structure. The structural changes of cyt-c are particularly visible when the number of surfactant molecules increases above CMC. The heme group in all considered systems exhibited high structural stability, not influenced by the addition of surfactant molecules, thus confirming low and stable values of RMSD. In Fig. S5, the RMSD values for selected fragments of the cyt- $c$ protein are presented. As it can be seen, the addition of surfactants affects the enhanced mobility of specific fragments of the protein which is related to the structural changes of the polypeptide chain. Below CMC, DMM-11 and DMPM-11 molecules especially influence the fluctuation of 14-26 and 72-90 fragments. However, above CMC the impact strictly depends on the type of surfactant molecule. In the case of DMM-11, the major changes take place around 1-25 fragments of protein, whereas, the presence of DMPM-11 affects the structure of the 100-105 fragments. Such structural effects are strictly related with interactions of the surfactant molecules with the amino acids from the protein surface. The characteristics of such interactions were herein described by the number of contacts between cationic surfactant molecules and cyt-c.

Table 1

Average values of atomic contact for DMM-11 and DMPM-11 surfactants.

\begin{tabular}{|c|c|c|c|c|c|}
\hline \multicolumn{2}{|c|}{ Surfactant molecules } & \multicolumn{2}{|c|}{ Distance $4[\AA]$} & \multicolumn{2}{|c|}{ Distance 5 [Å] } \\
\hline & & Contacts & SD & Contacts & SD \\
\hline 8 DMM-11 & $7(87.5 \%)$ & 434 & 134 & 1294 & 357 \\
\hline 30 DMM-11 & $6(20 \%)$ & 496 & 127 & 1736 & 408 \\
\hline 8 DMPM-11 & $6(75 \%)$ & 482 & 133 & 1486 & 417 \\
\hline 30 DMPM-11 & $6(20 \%)$ & 473 & 99 & 1643 & 333 \\
\hline
\end{tabular}

Our results suggest that, in the case of the DMPM- 11 system, regardless of the number of surfactant molecules used in the simulation, the interactions occur mainly in the active center of protein (Table 1 ). In the case of DMM-11, eight molecules interact similarly, while 20-30 amino acid residues localized under the active center of heme play a dominant role in the contact surface with 30 molecules of DMM-11. The increase of the range of interactions increases only the number of contacts (about three times), not affecting the number of interacting molecules. The highest flexibility of cyt-c fragments was connected also with the presence of the largest number of protein-surfactant contacts. The high mobility of the 100-105 fragments of cyt-c in the presence of 30 molecules of DMPM-11 is not related to mutual interactions of subsystems but to the decrease of interactions with the 20-30 fragments of protein. As it can be observed in Figs. 5, 6, 7 and Fig. 8, some surfactant molecules can interact with the protein surface through their polar parts but, in most cases influence via a hydrophobic moiety. Above CMC, DMM-11 and DMPM-11 have the ability to self-assemble through hydrophobic interactions with cyt-c The structural flexibility of protein chains after addition of surfactants molecules, influences the structural properties of active site, where the heme molecule is located. The structural modifications were confirmed by the increase of the maximum values of SASA and SAV after addition of surfactant (Fig. 9 and Table S3). On the other hand, solvent accessible surface and volume slightly decreases when the number of DMPM-11 exceeds the CMC. The slight structural changes of the active site that were observed did not influence the stability of the heme. The earlier observations related with RMSD values confirm also the distributions of hydrogen bonds created by Trp59 and oxygen's from the heme side chain (Table 2). For all complexes considered during simulation, the existing hydrogen bond is stable and nearly $99 \%$ taken into account that conformations meet the requirements for such interactions. However, small discrepancies in acceptor donor distance distributions were observed. The increase of active site solvent accessible volume (SAV) observed for some complexes correlate with the increased amount of conformers characterized by higher values of hydrogen bond length.

\section{Conclusions}

The interactions between the two cationic lysosomotropic surfactants DMM-11 and DMPM-11 and cyt-c were studied using surface tension, fluorescence measurements, $C D$ spectroscopy and molecular dynamic simulations. The multiple spectroscopic methods indicated that both cationic surfactants can interact with cyt-c and that the major binding mechanisms involved are electrostatic and hydrophobic interactions. Both single-chain surfactants were found to be able to interact with cyt-c to form hemichrome. The surfactants turn the secondary structure of cyt-c loose due to the hydrophobic interactions and changes in the mobility of specific fragments of the protein, thus leading to its conversion into the $\beta$-sheet structure. Based on the molecular dynamic simulations and computation analysis, we propose for first time a novel theoretical model at the atomic level for the interactions between cyt-c and the QACs DMM-11 and DMPM-11. Overall, this study provides a complete description of the nature of interactions between lysosomotropic surfactants and cyt-c which is useful to design drug delivery and medical applications.

\section{Conflict of interest}

Authors declare no conflict of interest.

\section{Acknowledgments}

This work was supported by the Polish-Portugal Executive Program for years 2017-2018 sponsored by the Polish Ministry of Science and Higher Education and by the Portuguese Foundation for Science and Technology (FCT). Additionally, FCT supported the study under the 
$1 \mathrm{~ns}$

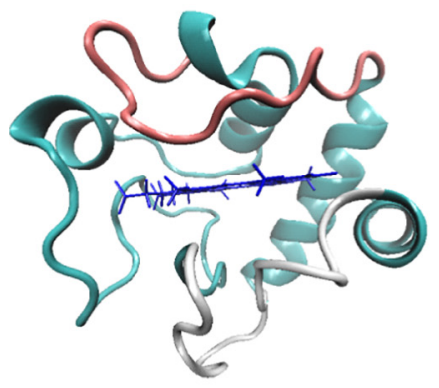

$100 \mathrm{~ns}$

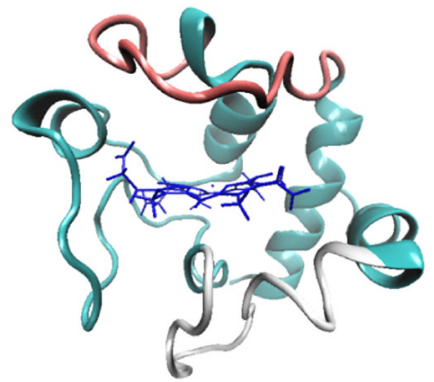

200 ns

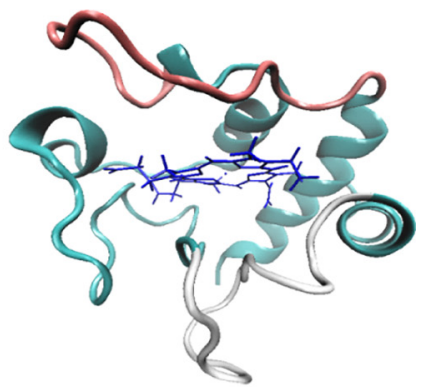

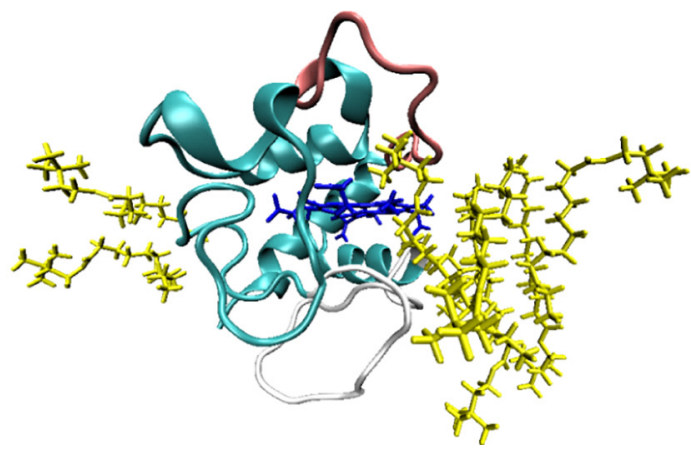

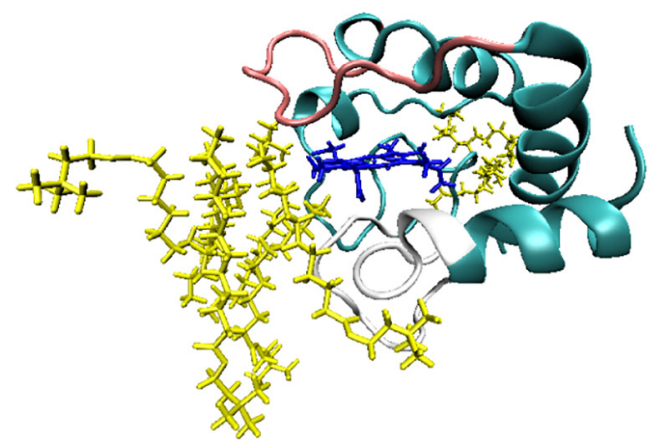

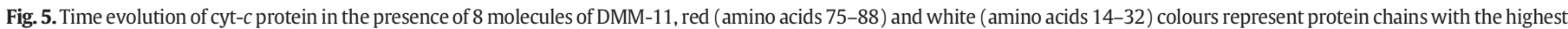
flexibility during molecular dynamics simulation.

scope of the strategic funding of UID/BIO/04469/2013 unit and COMPETE 2020 (POCI-01-0145-FEDER-006684). T. Janek was supported by the National Science Centre, Poland, projects 2017/26/E/NZ9/00975 and 2018/02/X/NZ6/02201. L. R. Rodrigues acknowledges FCT for the grant SFRH/BSAB/142873/2018. E. J. Gudiña was supported by the grant UMINHO/BPD/39/2015 funded by FCT under the scope of the project
1 ns

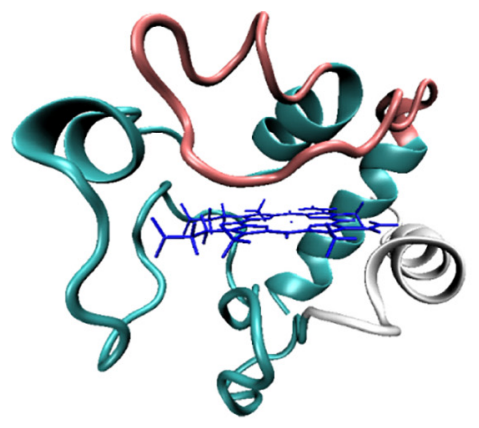

$100 \mathrm{~ns}$

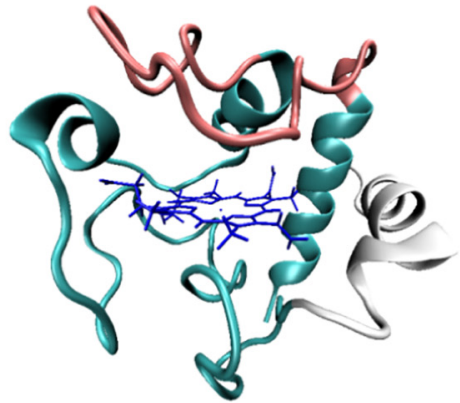

200 ns

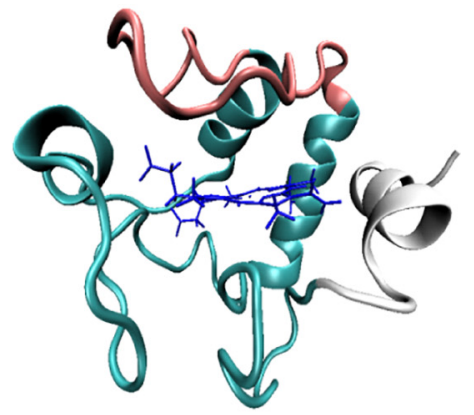

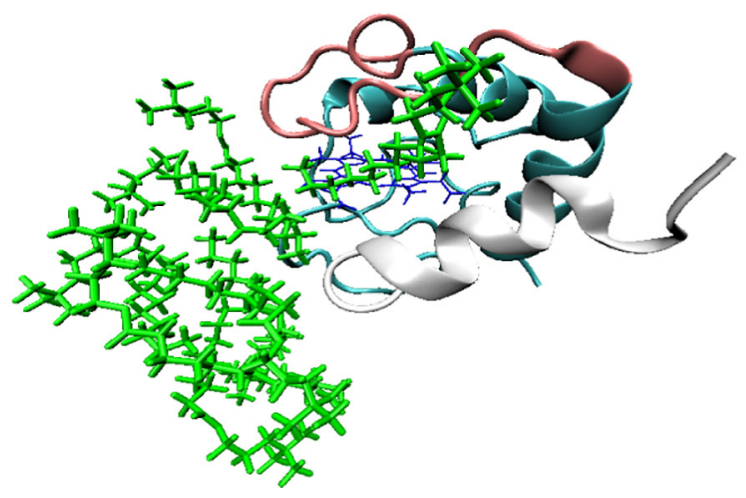

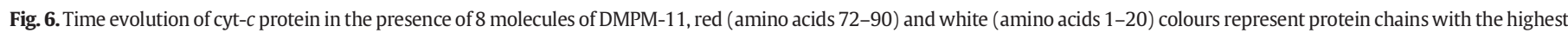
flexibility during molecular dynamics simulation. 
1 ns

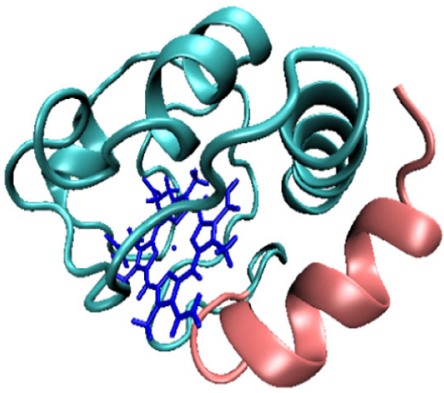

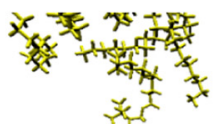

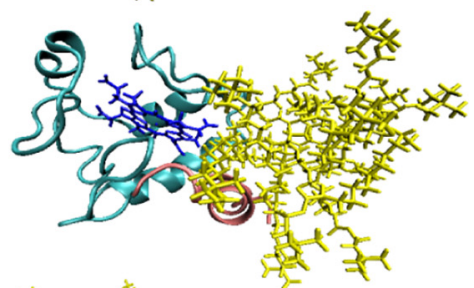

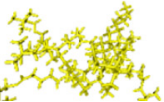

$100 \mathrm{~ns}$
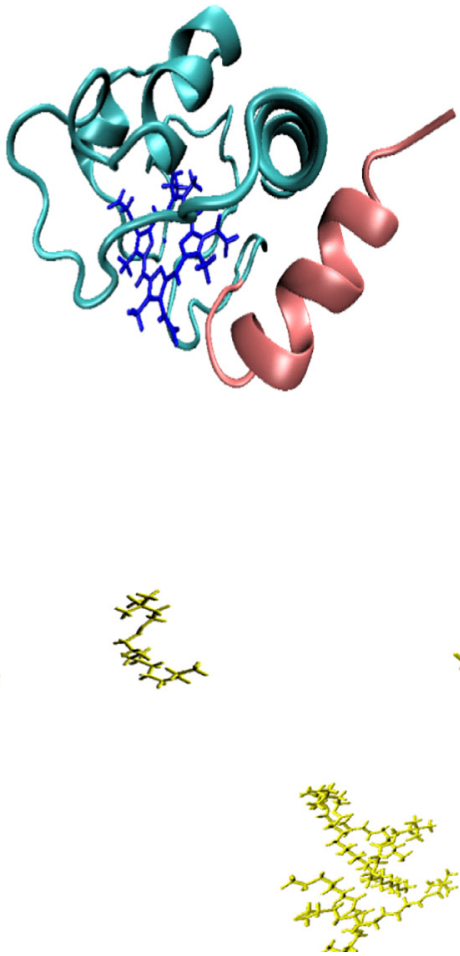

200 ns

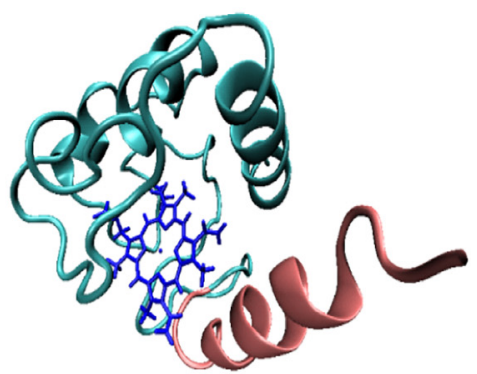

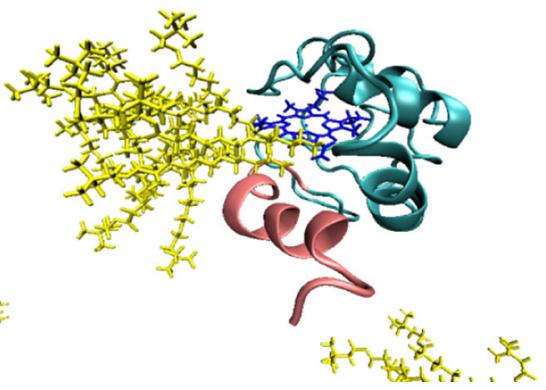

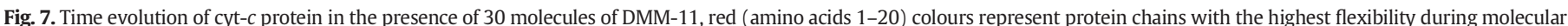
dynamics simulation.

1 ns
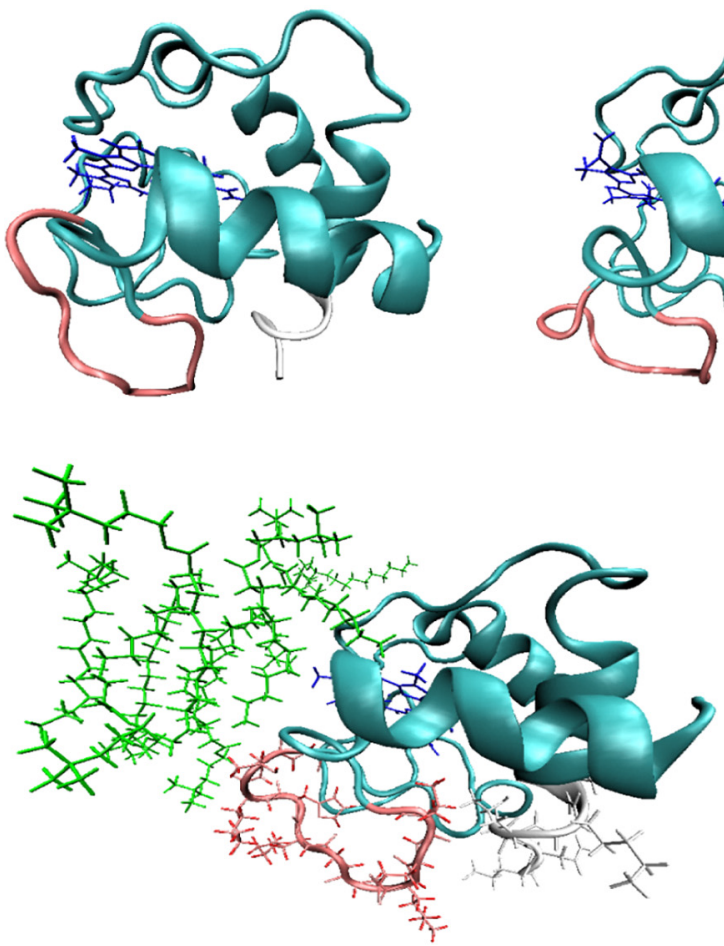

$100 \mathrm{~ns}$
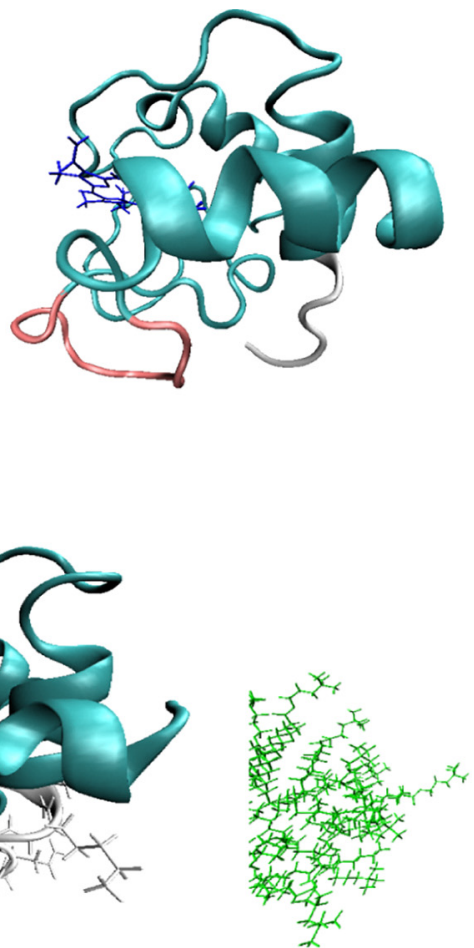

200 ns
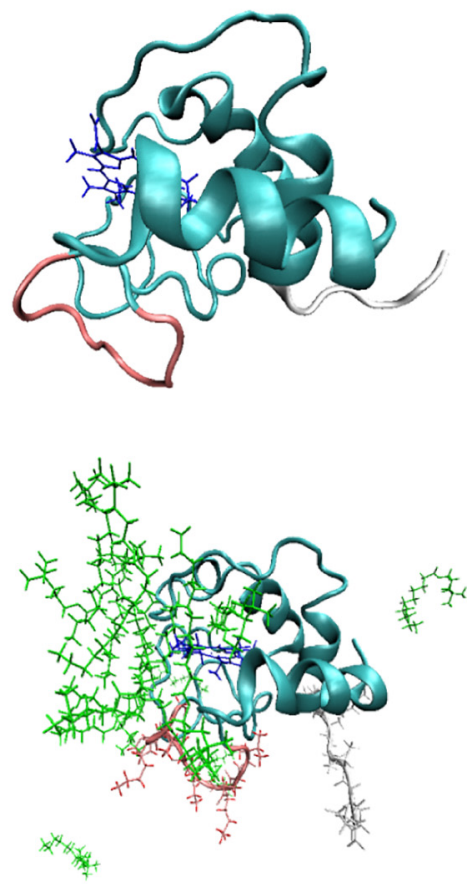

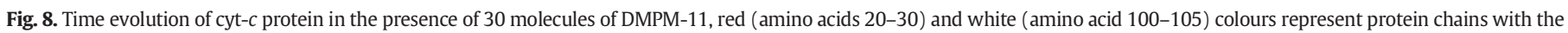
highest flexibility during molecular dynamics simulation. 

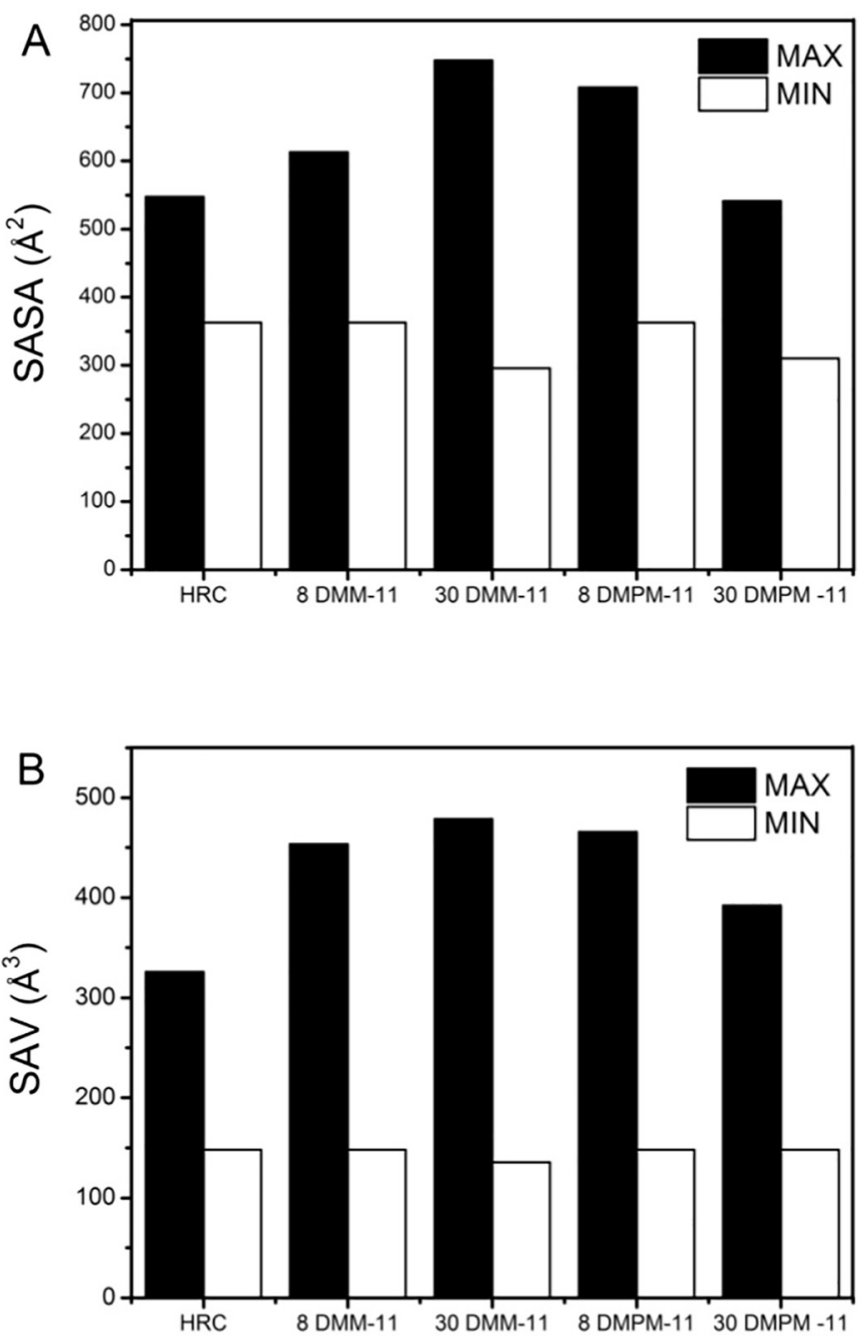

Fig. 9. Bar diagram of calculated values of (A) accessible surface area $\left[\AA^{2}\right]$, (B) accessible surface volume $\left[\AA^{3}\right]$.

UID/BIO/04469/2013. The authors would like to kindly thank Dr. Jacek Łuczyński from the Wroclaw University of Science and Technology for the opportunity to carry out research using the compounds that he synthesized.

\section{Appendix A. Supplementary data}

Supplementary data to this article can be found online at https://doi. org/10.1016/j.ijbiomac.2019.01.024.

Table 2

Characteristics of the hydrogen bond created by Trp59 and the heme molecule.

\begin{tabular}{llllll}
\hline \multirow{2}{*}{ Distance $[\AA]$} & \multicolumn{5}{l}{ Population [\%] } \\
\cline { 2 - 6 } & Cyt-c & Cyt-c & Cyt-c & Cyt-c & Cyt-c \\
& & 8 DMM-11 & 8 DMPM-11 & 30 DMM-11 & 30 DMPM-11 \\
\hline 1.75 & 20.4 & 13.8 & 14.0 & 9.9 & 10.4 \\
2 & 47.9 & 46.2 & 44.9 & 44.6 & 44.3 \\
2.25 & 19.9 & 27.0 & 30.1 & 33.7 & 31.0 \\
2.5 & 6.5 & 8.6 & 8.6 & 8.8 & 9.6 \\
2.75 & 1.8 & 3.0 & 1.5 & 1.6 & 2.1 \\
3 & 0.7 & 0.7 & 0.6 & 0.5 & 0.9 \\
\hline
\end{tabular}

\section{References}

[1] S.K. Chaturvedi, J.M. Khan, M.K. Siddiqi, P. Alam, R.H. Khan, Comparative insight into surfactants mediated amyloidogenesis of lysozyme, Int. J. Biol. Macromol. 83 (2016) 315-325, https://doi.org/10.1016/j.ijbiomac.2015.11.053.

[2] M. Kumari, J.K. Maurya, U.K. Singh, A.B. Khan, M. Ali, P. Singh, R. Patel, Spectroscopic and docking studies on the interaction between pyrrolidinium based ionic liquid and bovine serum albumin, Spectrochim. Acta A Mol. Biomol. Spectrosc. 124 (2014) 349-356, https://doi.org/10.1016/j.saa.2014.01.012.

[3] M. Kumari, U.K. Singh, I. Beg, A.M. Alanazi, A.A. Khan, R. Patel, Effect of cations and anions of ionic liquids on the stability and activity of lysozyme: concentration and temperature effect, J. Mol. Liq. 272 (2018) 253-263, https://doi.org/10.1016/j. molliq.2018.09.075.

[4] T. Janek, J. Czyżnikowska, E.J. Łuczyński, L.R. Gudiña, J. Rodrigues, Gałęzowska, Physicochemical study of biomolecular interactions between lysosomotropic surfactants and bovine serum albumin, Colloids Surf. B: Biointerfaces 159 (2017) 750-758, https://doi.org/10.1016/j.colsurfb.2017.08.046.

[5] S. Ghosh, J. Dey, Interaction of bovine serum albumin with $\mathrm{N}$-acyl amino acid based anionic surfactants: effect of head-group hydrophobicity, J. Colloid Interface Sci. 458 (2015) 284-292, https://doi.org/10.1016/j.jcis.2015.07.064.

[6] A. Iovescu, A. Bəran, G. Stîngə, A.R. Cantemir-Leonties, M.E. Maxim, D.F. Anghel, A combined binding mechanism of nonionic ethoxylated surfactants to bovine serum albumin revealed by fluorescence and circular dichroism, J. Photochem. Photobiol. B Biol. 153 (2015) 198-205, https://doi.org/10.1016/j.jphotobiol.2015. 09.021.

[7] T.W. Randolph, L.S. Jones, Surfactant-protein interactions, Pharm. Biotechnol. 13 (2002) 159-175, https://doi.org/10.1007/978-1-4615-0557-0.

[8] N. Gull, J.M. Khan, R.H. Khan Rukhsana, Spectroscopic studies on the gemini surfactant mediated refolding of human serum albumin, Int. J. Biol. Macromol. 102 (2017) 331-335, https://doi.org/10.1016/j.ijbiomac.2017.03.134.

[9] P. Qu, H. Lu, S. Yan, Z. Lu, Influences of cationic, anionic, and nonionic surfactants on alkaline-induced intermediate of bovine serum albumin, Int. J. Biol. Macromol. 46 (2010) 91-99, https://doi.org/10.1016/j.ijbiomac.2009.10.012.

[10] M.A. Branco, L. Pinheiro, C. Faustino, Amino acid-based cationic gemini surfactantprotein interactions, Colloids Surf. A Physicochem. Eng. Asp. 480 (2015) 105-112, https://doi.org/10.1016/j.colsurfa.2014.12.022.

[11] I.A. Bhat, W.F. Bhat, M. Akram, Kabir-ud-Din, Interaction of a novel twin-tailed oxydiester functionalized surfactant with lysozyme: spectroscopic and computational perspective, Int. J. Biol. Macromol. 109 (2018) 1006-1011, https://doi.org/10.1016/ j.ijbiomac.2017.11.085.

[12] U.K. Singh, M. Kumari, S.H. Khan, H.B. Bohidar, R. Patel, Mechanism and dynamics of long-term stability of cytochrome $c$ conferred by long-chain imidazolium ionic liquids at low concentration, ACS Sustain. Chem. Eng. 6 (2018) 803-815, https://doi. org/10.1021/acssuschemeng.7b03168.

[13] M. ud din Parray, N. Maurya, F. Ahmad Wani, M.S. Borse, N. Arfin, M. Ahmad Malik, R. Patel, Comparative effect of cationic gemini surfactant and its monomeric counterpart on the conformational stability of phospholipase A2, J. Mol. Struct. 1175 (2019) 49-55, https://doi.org/10.1016/j.molstruc.2018.07.078.

[14] M. Hu, X. Wang, H. Wang, Y. Chai, Y. He, G. Song, Fluorescence spectroscopic studies on the interaction of Gemini surfactant 14-6-14 with bovine serum albumin, Luminescence 27 (2012) 204-210, https://doi.org/10.1002/bio.1333.

[15] E. Oblak, T.M. Lachowicz, J. Luczynski, S. Witek, N. Lysosomotropic, N- dimethyl alpha-aminoacid $N$-alkyl esters and their quaternary ammonium salts as plasma membrane and mitochondrial ATPases inhibitors, Cell. Mol. Biol. Lett. 7 (2002) 1121-1129http://www.ncbi.nlm.nih.gov/pubmed/12511979.

[16] E. Oblak, A. Krasowska, The influence of organic nitrogen compounds on melanoma, bacterial, and fungal cells, Adv. Clin. Exp. Med. 19 (2010) 65-75.

[17] T. Jain, P.R. Muktapuram, K. Sharma, O. Ravi, G. Pant, K. Mitra, S.R. Bathula, D. Banerjee, Biofilm inhibition and anti-Candida activity of a cationic lipo-benzamide molecule with twin-nonyl chain, Bioorg. Med. Chem. Lett. 28 (2018) 1776-1780, https://doi.org/10.1016/j.bmcl.2018.04.024.

[18] L.Y. Zakharova, T.N. Pashirova, A.R. Fernandes, S. Doktorovova, C. Martins-Gomes, A.M. Silva, E.B. Souto, Self-assembled quaternary ammonium surfactants for pharmaceuticals and biotechnology, Org. Mater. as Smart Nanocarriers Drug Deliv. 2018, pp. 601-618, https://doi.org/10.1016/B978-0-12-813663-8.00014-2.

[19] R.A. Allen, M.C. Jennings, M.A. Mitchell, S.E. Al-Khalifa, W.M. Wuest, K.P.C. Minbiole, Ester- and amide-containing multiQACs: exploring multicationic soft antimicrobial agents, Bioorg. Med. Chem. Lett. 27 (2017) 2107-2112, https://doi.org/10.1016/j. bmcl.2017.03.077.

[20] D. Wang, C. Richter, A. Rühling, S. Hüwel, F. Glorius, H.J. Galla, Anti-tumor activity and cytotoxicity in vitro of novel 4,5-dialkylimidazolium surfactants, Biochem. Biophys. Res. Commun. 467 (2015) 1033-1038, https://doi.org/10.1016/j.bbrc. 2015.10.015.

[21] J.P. Shrestha, C. Baker, Y. Kawasaki, Y.P. Subedi, N.N. Vincent de Paul, J.Y. Takemoto C.W.T. Chang, Synthesis and bioactivity investigation of quinone-based dimeric cationic triazolium amphiphiles selective against resistant fungal and bacterial pathogens, Eur. J. Med. Chem. 126 (2017) 696-704, https://doi.org/10.1016/j.ejmech. 2016.12.008.

[22] Y. Pan, Z. Wang, D. Shao, H. Zheng, Y. Chen, X. Zheng, M. Zhang, J. Li, F. Li, L. Chen, CTAB induced mitochondrial apoptosis by activating the AMPK-p53 pathway in hepatocarcinoma cells, Toxicol. Res. 4 (2015) 1359-1365, https://doi.org/10.1039/ c4tx00227j.

[23] G.C. Mills, Cytochrome c: gene structure, homology and ancestral relationships, J. Theor. Biol. 152 (1991) 177-190, https://doi.org/10.1016/S0022-5193(05)80451-5.

[24] S. Elmore, Apoptosis: a review of programmed cell death, Toxicol. Pathol. 35 (2007) 495-516, https://doi.org/10.1080/01926230701320337. 
[25] A. Patriarca, T. Eliseo, F. Sinibaldi, M.C. Piro, R. Melis, M. Paci, D.O. Cicero, F. Polticelli, R. Santucci, L. Fiorucci, ATP acts as a regulatory effector in modulating structural transitions of cytochrome c: implications for apoptotic activity, Biochemistry 48 (2009) 3279-3287, https://doi.org/10.1021/bi801837e.

[26] J. Łuczyński, Aminoethylesters of Fatty Acids as Lysosomotropic Substances, Wrocław University of Science and Technology, 2000.

[27] G. Para, J. Łuczyński, J. Palus, E. Jarek, K.A. Wilk, P. Warszyński, Hydrolysis driven surface activity of esterquat surfactants, J. Colloid Interface Sci. 465 (2016) 174-182 https://doi.org/10.1016/j.jcis.2015.11.056.

[28] C. Huh, S.G. Mason, Rigorous theory of ring tensiometry, Colloid Polym. Sci. 253 (1975) 566-580

[29] R. Patel, M. ud din Parray, U.K. Singh, A. Islam, P. Venkatesu, S. Singh, H.B. Bohidar, Effect of 1,4-bis(3-dodecylimidazolium-1-yl) butane bromide on channel form of gramicidin vesicles, Colloids Surf. A Physicochem. Eng. Asp. 508 (2016) 150-158, https://doi.org/10.1016/j.colsurfa.2016.08.058.

[30] J.R. Lakowicz, Principles of fluorescence spectroscopy principles of fluorescence, Spectroscopy (2006)https://doi.org/10.1007/978-0-387-46312-4

[31] A.K.D.A. Case, V. Babin, J.T. Berryman, R.M. Betz, Q. Cai, D.S. Cerutti, T.E. Cheatham II, T.A. Darden, R.E. Duke, H. Gohlke, A.W. Goetz, S. Gusarov, N. Homeyer, P. Janowski, J. Kaus, I. Kolossváry, X. W., P.A.K.T.S. Lee, S. LeGrand, T. Luchko, R. Luo, B. Madej, K.M. Merz, F. Paesani, D.R. Roe, A. Roitberg, C. Sagui, R. Salomon-Ferrer, G. Seabra, C.L. Simmerling, W. Smith, J. Swails, R.C. Walker, J. Wang, R.M. Wolf, Amber14 Reference Manual, 2014https://doi.org/10.1007/s13398-014-0173-7.2.

[32] G.W. Bushnell, G.V. Louie, G.D. Brayer, High-resolution three-dimensional structure of horse heart cytochrome c, J. Mol. Biol. 214 (1990) 585-595, https://doi.org/10. 1016/0022-2836(90)90200-6.

[33] C.I. Bayly, P. Cieplak, W.D. Cornell, P.A. Kollman, A well-behaved electrostatic potential based method using charge restraints for deriving atomic charges: the RESP model, J. Phys. Chem. 97 (1993) 10269-10280, https://doi.org/10.1021/j100142a004.

[34] D.A. Giammona, D.A. Case, C. Bayly, Force Field Modifications for all-Atom Heme 1995.

[35] W. Humphrey, A. Dalke, K. Schulten, VMD: visual molecular dynamics, J. Mol. Graph. 14 (1996) 33-38, https://doi.org/10.1016/0263-7855(96)00018-5.
[36] J. Dundas, Z. Ouyang, J. Tseng, A. Binkowski, Y. Turpaz, J. Liang, CASTp: computed atlas of surface topography of proteins with structural and topographical mapping of functionally annotated residues, Nucleic Acids Res. 34 (2006)https://doi.org/10. 1093/nar/gkl282.

[37] D. Wu, G. Xu, Y. Feng, Y. Li, Aggregation behaviors of gelatin with cationic gemini surfactant at air/water interface, Int. J. Biol. Macromol. 40 (2007) 345-350, https://doi. org/10.1016/j.ijbiomac.2006.09.004.

[38] N. Nishikido, T. Takahara, H. Kobayashi, M. Tanaka, Interaction between hydrophilic proteins and nonionic detergents studied by surface tension measurements, Bull. Chem. Soc. Jpn. 55 (1982) 3085-3088, https://doi.org/10.1246/bcsj.55.3085.

[39] A. Zaragoza, J.A. Teruel, F.J. Aranda, A. Marqués, M.J. Espuny, Á. Manresa, A. Ortiz, Interaction of a Rhodococcus sp. Trehalose lipid biosurfactant with model proteins: thermodynamic and structural changes, Langmuir 28 (2012) 1381-1390, https:// doi.org/10.1021/la203879t.

[40] D. Hamada, M. Hoshino, M. Kataoka, Y. Goto, A.L. Fink Intermediate conformational states of Apocytochrome c, Biochemistry 32 (1993) 10351-10358, https://doi.org/ 10.1021/bi00090a010.

[41] T.D. Gauthier, E.C. Shane, W.F. Guerin, W.R. Seitz, C.L. Grant, Fluorescence quenching method for determining equilibrium constants for polycyclic aromatic hydrocarbons binding to dissolved humic materials, Environ. Sci. Technol. 20 (1986) 1162-1166, https://doi.org/10.1021/es00153a012.

[42] U. Ahluwalia, S.M. Nayeem, S. Deep, The non-native conformations of cytochrome c in sodium dodecyl sulfate and their modulation by ATP, Eur. Biophys. J. 40 (2011) 259-271, https://doi.org/10.1007/s00249-010-0643-6.

[43] J.P. Hennessey, W.C. Johnson, Information content in the circular dichroism of proteins, Biochemistry 20 (1981) 1085-1094, https://doi.org/10.1021/bi00508a007.

[44] V. Jafari Azad, S. Kasravi, H. Alizadeh Zeinabad, M. Memar Bashi Aval, A.A. Saboury, A. Rahimi, M. Falahati, Probing the conformational changes and peroxidase-like activity of cytochrome c upon interaction with iron nanoparticles, J. Biomol. Struct. Dyn. 35 (2017) 2565-2577, https://doi.org/10.1080/07391102.2016.1222972. 